\title{
Zeeman coupling and screening corrections to skyrmion excitations in graphene
}

\author{
Wenchen Luo $^{1}$ and R. Côté ${ }^{1}$ \\ ${ }^{1}$ Département de physique, Université de Sherbrooke, Sherbrooke, Québec, J1K 2R1, Canada
}

(Dated: August 12, 2018)

\begin{abstract}
At half filling of the fourfold degenerate Landau levels $|n| \geq 1$ in graphene, the ground states are spin polarized quantum Hall states that support spin skyrmion excitations for $|n|=1,2,3$. Working in the Hartree-Fock approximation, we compute the excitation energy of an unbound spin skyrmionantiskyrmion excitation as a function of the Zeeman coupling strength for these Landau levels. We find for both the bare and screened Coulomb interactions that the spin skyrmion-antiskyrmion excitation energy is lower than the excitation energy of an unbound spin $1 / 2$ electron-hole pair in a finite range of Zeeman coupling in Landau levels $|n|=1,2,3$. This range decreases rapidly for increasing Landau level index and is extremely small for $|n|=3$. For valley skyrmions which should be present at $1 / 4$ and $3 / 4$ fillings of the Landau levels $|n|=1,2,3$, we show that screening corrections are more important in the latter case. It follows that an unbound valley skyrmionantiskyrmion excitation has lower energy at $3 / 4$ filling than at $1 / 4$. We compare our results with recent experiments on spin and valley skyrmion excitations in graphene.
\end{abstract}

PACS numbers: 73.50.Fq,72.10.-d,73.21.-b

\section{INTRODUCTION}

The energy of the Landau levels in graphene in a transverse magnetic field $\mathbf{B}=-B \widehat{\mathbf{z}}$ is given by

$$
E_{s, n}^{0}=\operatorname{sgn}(n) \sqrt{|n|} \frac{\sqrt{2} \hbar v_{F}}{\ell}-\frac{1}{2} \operatorname{sg} \mu_{B} B,
$$

where $n=0, \pm 1, \pm 2, \ldots$ is the Landau level index, $s= \pm 1$ is the spin index, $\ell=\sqrt{\hbar c / e B}$ is the magnetic length, $v_{F}$ is the Fermi velocity, $g=2$ is the Landé factor and $\mu_{B}$ is the Bohr magneton. Because the Zeeman energy is very small in comparison with the kinetic energy, each Landau level is usually considered as being fourfold degenerate when counting spin and valley $\left(K_{ \pm}\right)$degrees of freedom. Experimentally, this kinetic energy quantization leads to the anomalous Hall sequence $\frac{1}{}$

$$
\sigma_{x y}= \pm \frac{4 e^{2}}{h}\left(n+\frac{1}{2}\right)
$$

in the Hall conductivity and so to quantum Hall plateaus at filling factors $\nu= \pm 4\left(n+\frac{1}{2}\right)$.

In experiments ${ }^{2}$ on very high quality graphene samples fabricated on hexagonal boron nitride (hBN) substrates, it is possible to resolve the quantum Hall plateaus at all integer filling factors, i.e. $\sigma_{x y}=m e^{2} / h$ with $|m| \geq 0^{\underline{3}}$, and to see an insulating state developing at filling factor $\nu=0$. These experiments allow the study of the nature of the quantum Hall ground states of the chiral twodimensional electron gas (C2DEG) as well as the nature of their charged excitations. In Ref. 2, it was shown that the ground states are maximally spin polarized at filling factors $\nu=-4,-8,-12$ while the ground state at $\nu=0$ is not. Moreover, the charged excitations were found to be spin texture excitations at half filling of Landau levels $n=-1,-2$ in some range of Zeeman coupling. Valley skyrmions were also detected and studied at filling factors $\nu=-3,-5$ where the ground state is valley polarized.
Theoretically, a calculation based on the nonlinear $\sigma$ model ( $N L \sigma$ model) that is valid at zero Zeeman coupling shows that, in graphene, the transport gap should be due to spin texture i.e. spin skyrmion excitations 4,5 at half filling of Landau levels $|n|=1,2,3^{6}$ and to valley skyrmions at $1 / 4$ and $3 / 4$ filling of these same levels. Because there are no symmetry-breaking terms associated with the two valleys (i.e. no equivalent Zeeman coupling), the $N L \sigma$ model calculation of Ref. 6 describes the valley skyrmions very well. In graphene, spin skyrmions thus persist to higher Landau levels than in a conventional semiconductor two-dimensional electron gas (2DEG). Indeed, in a semiconductor 2DEG, skyrmions are the lowest-energy charged excitations in $n=0$ at filling factor $\nu=1$ only (when the width of the quantum well is neglected) $\underline{7}$. . In higher Landau levels, the transport gap is due to unbound electron-hole pairs ${ }^{9}$. The same conclusion concerning skyrmions in graphene was reached using the density matrix renormalization group (DMRG) method for $n=0,1,2$. For $n=3$, the skyrmion-antiskyrmion (S-aS) pair and electron-hole pair energies are very close and it was not possible to stabilize a skyrmion solution with the DMRG method 10 . Exact diagonalization studies of valley skyrmions have also been done in Ref. 11. Crystals of valley skyrmions have been shown to be the ground state of the C2DEG around quarter filling of the $n=0,1$ Landau levels $\frac{12}{2}$. A theoretical study of the possible entanglement between the spin and valley degrees of freedom in graphene which could lead to $\mathrm{CP}^{3}$ skyrmions was done in Ref. 13. This work did not include a calculation of the behavior of the transport gap with Zeeman coupling however.

The energy functional of the $N L \sigma$ model in brokensymmetry quantum Hall ferromagnetic states contains a gradient term that originates from the exchange part of the Coulomb interaction. The topological solitons of this model can be determined exactly 14 . The gradient term being scale invariant in two dimensions, the energy 
of these solutions is independent of their size. When a finite Zeeman coupling is considered, two more terms must be added to the $N L \sigma$ model energy functional: a Zeeman coupling which favors small skyrmions and an electrostatic self-interaction energy which favors large skyrmions. These two terms compete together to determine the optimal size and energy of a skyrmion as well as its density profile and spin texture.

In this paper, we study the energetics of spin skyrmions in graphene in the half-filled Landau levels $|n|=1,2,3$ and valley-skyrmions at $1 / 4$ and $3 / 4$ fillings where the ground state is spin and valley polarized. For spin skyrmions, we extend the calculation of the $N L \sigma$ model, valid at zero Zeeman coupling, to finite Zeeman coupling by using a Green's function approach. The equation of motion of the Green's function is derived in the Hartree-Fock approximation and in the symmetric gauge. This derivation leads to a set of coupled self-consistent equations for the angular momentum components of the skyrmion wave function which must be solved numerically using an iterative procedure. Our approach is equivalent to the canonical transformation method used earlier in the study of skyrmion in a semiconductor 2DEG 15 . Our method works well with finite-size skyrmions but cannot deal with very large skyrmions which are obtained at small Zeeman coupling since large skyrmions require a large number of angular momentum components for their description.

We compute the unbound S-aS pair energy, $\Delta_{S-a S}$, and compare it with the energy to make an unbound electron-hole pair $\Delta_{e-h}$. The transport gap is determined by the lowest of these two energies. We find that spin-texture excitations are the lowest-energy excitations at half-filling in a small range of Zeeman coupling for $n=1,2$ and that this range decreases rapidly with increasing Landau level index. According to the $N L \sigma$ model, spin skyrmions are the lowest-energy excitations at zero Zeeman coupling also in $n=3$. With the limitations of our method, however, we cannot find spin skyrmions at finite Zeeman coupling for $n=3$. It follows that the Zeeman coupling range where they are the lowest-energy excitations must be very small.

It is straightforward to modify our method to include screening of the Coulomb interaction. The Coulomb matrix elements that enter the equation of motion for the Green's functions are evaluated using a dielectric function computed in the random-phase approximation (RPA). For spin skyrmions, we find that screening decreases substantially the transport gaps $\Delta_{N L \sigma M}, \Delta_{S-a S}$ and $\Delta_{e-h}$ as well as the critical Zeeman coupling for the transition between $\Delta_{S-a S}$ and $\Delta_{e-h}$. Nevertheless, the skyrmion scenario still prevails for $n=1,2$ and for $n=3$ in a very small range of Zeeman coupling.

For valley skyrmions, there is no symmetry-breaking term equivalent to the Zeeman coupling so that the transport gap can be computed using the $N L \sigma M$. Our results show that screening corrections are more important at $3 / 4$ filling than at $1 / 4$ so that the transport gap due to unbound valley S-aS excitations is lower in the former case.

This paper is organized in the following way. In Sec. II, we introduce the spinor non-interacting electronic states of graphene in a magnetic field using the symmetric gauge. Sec. III summarizes the Hartree-Fock approximation to the electron-electron interaction and presents the assumptions necessary to derive the two-level system that is the starting point of our work. Sec. IV contains a description of the quasiparticle (electron and hole) and skyrmion excitations. The Green's function formalism is discussed in Sec. V. The numerical results are presented and discussed in Sec. VI for the unscreened Coulomb interaction and in Sec. VII for the screened interaction. We conclude in Sec. VIII.

\section{TIGHT-BINDING HAMILTONIAN AND EIGENSTATES OF THE NON-INTERACTING CHIRAL 2DEG}

Graphene has a honeycomb lattice structure that can be described as an hexagonal Bravais lattice with a lattice constant $a_{0}=2.46 \AA$ and a basis of two carbon atoms $A$ and $B^{1}$. Each carbon atom contributes one electron to the two $\pi$ bands. These electrons form a C2DEG. In the sublattice basis $(A, B)$, the Hamiltonian in a transverse magnetic field $\mathbf{B}=-B \widehat{\mathbf{z}}$ and in the continuum approximation (i.e. for small energy with respect to the Dirac points) is given by

$$
\mathcal{H}_{\alpha}=\alpha \frac{\sqrt{2} \hbar v_{F}}{\ell}\left(\begin{array}{cc}
0 & a^{\mp} \\
a^{ \pm} & 0
\end{array}\right)
$$

where $\alpha= \pm$ is the valley index for the two nonequivalent valleys $\mathbf{K}_{\alpha}=\alpha(2 / 3,0)\left(2 \pi / a_{0}\right)$ in the Brillouin zone and $v_{F}=\sqrt{3} \gamma_{0} a_{0} / 2 \hbar$ is the Fermi velocity with $\gamma_{0}=3.12$ $\mathrm{eV}$ the hopping energy between nearest-neighbors carbon atoms. The operators $a^{+}=a^{\dagger}, a^{-}=a$ are the ladder operators for the one-dimensional harmonic oscillator. In Eq. (3), the upper(lower) sign is for the $\alpha=+(-)$ valley.

The Landau level spectrum of $\mathcal{H}_{\alpha}$ is given by

$$
E_{s, n}^{0}=\operatorname{sgn}(n) \sqrt{|n|} \frac{\sqrt{2} \hbar v_{F}}{\ell}-\frac{1}{2} s \Delta_{Z} .
$$

In Eq. (44), we have added a Zeeman coupling $\Delta_{Z}=$ $g \mu_{B} B$ to $\mathcal{H}_{\alpha}$. The Landau level index $n=0, \pm 1, \pm 2, \ldots$ takes both positive and negative values. In the absence of Zeeman coupling, each Landau level is fourfold degenerate when counting valley and spin degrees of freedom. In addition, each Landau level has the macroscopic orbital degeneracy $N_{\varphi}=S / 2 \pi \ell^{2}$ where $S$ is the C2DEG area.

The eigenstates of $\mathcal{H}_{\alpha}$ are spinors in the sublattice basis $(A, B)$. For $n \neq 0$, these spinors are for the two valleys 
and for a given spin orientation, given by

$$
\begin{aligned}
& |n, m, \alpha=+\rangle=\frac{1}{\sqrt{2}}\left(\begin{array}{c}
\operatorname{sgn}(n)|| n|-1, m\rangle \\
|| n|, m\rangle
\end{array}\right), \\
& |n, m, \alpha=-\rangle=\frac{1}{\sqrt{2}}\left(\begin{array}{c}
|| n|, m\rangle \\
-\operatorname{sgn}(n)|| n|-1, m\rangle
\end{array}\right)
\end{aligned}
$$

while for $n=0$, the eigenspinors are given by

$$
\begin{aligned}
& |0, m, \alpha=+\rangle=\left(\begin{array}{c}
0 \\
|0, m\rangle
\end{array}\right), \\
& |0, m, \alpha=-\rangle=\left(\begin{array}{c}
|0, m\rangle \\
0
\end{array}\right) .
\end{aligned}
$$

There is a direct correspondence between valley and sublattice indices in Landau level $n=0$.

The spinors in Eqs. (5, 8 ) are written in the symmetric gauge $\mathbf{A}=(B y / 2,-B x / 2)$, where the quantum number $m=0,1,2,3, \ldots$ is associated with the angular momentum by the relation

$$
L_{z}|n, m\rangle=(m-n) \hbar|n, m\rangle .
$$

The states $|n, m\rangle$ are simply the eigenstates of a conventional (non chiral) 2DEG in a magnetic field. Because of the symmetry of the skyrmion charged excitations, the symmetric gauge is the most convenient one.

In real space, the corresponding wave functions are given by ${ }^{16}$

$$
\begin{aligned}
\varphi_{n, m}(\mathbf{r}) \equiv & \langle\mathbf{r} \mid n, m\rangle \\
= & B_{n, m} e^{i(m-n) \phi}\left(\frac{r}{\ell}\right)^{|m-n|} e^{-\frac{r^{2}}{4 \ell^{2}}} \\
& \times L_{\frac{n+m}{2}-\frac{|n-m|}{2}}^{|m-n|}\left(\frac{r^{2}}{2 \ell^{2}}\right),
\end{aligned}
$$

where $\phi$ is the angle between the vector $\mathbf{r}$ and the $x$ axis, $L_{n}^{m}(x)$ is a generalized Laguerre polynomial and the normalization constant is given by

$$
B_{n, m}=\frac{C_{n, m}(-i)^{n}}{\sqrt{2^{|m-n|+1} \pi \ell^{2}}} \sqrt{\frac{\left(\frac{n+m}{2}-\frac{|n-m|}{2}\right) !}{\left(\frac{n+m}{2}+\frac{|n-m|}{2}\right) !}}
$$

with $C_{n, m}=1$ for $m \leq n$ and $C_{n, m}=(-1)^{m-n}$ for $m>n$.

\section{HARTREE-FOCK APPROXIMATION TO THE INTERACTING CHIRAL 2DEG}

In this paper, we consider the situation where the quartet of states in Landau level $n$ is partially filled and levels $n^{\prime}<n$ are completely filled. We make the approximation of considering the filled levels as inert so that we can ignore them altogether. We thus neglect Landau level mixing. It must be kept in mind, however, that in graphene the difference in the kinetic energy between the first two
Landau levels $E_{C}=\sqrt{2} \hbar v_{F} / \ell=3.67 \times 10^{-2} \sqrt{B} \mathrm{eV}=$ $426 \sqrt{B} \mathrm{~K}$ is of the order of the Coulomb interaction $e^{2} / \kappa \ell=2.25 \times 10^{-2} \sqrt{B} \mathrm{eV}=261 \sqrt{B} \mathrm{~K}$ (for $\kappa=2.5$ appropriate for graphene on hexagonal boron nitride and $B$ in Tesla) and Landau level mixing may be important. The Zeeman energy $\Delta_{Z}=g \mu_{B} B=1.16 \times 10^{-4} B$ $\mathrm{eV}=1.34 B \mathrm{~K}$.

We need to consider the Coulomb interaction between electrons in level $n$ which is given in second-quantization by

$$
\begin{aligned}
V= & \frac{1}{2} \sum_{\alpha, \beta, s, s^{\prime}} \int d \mathbf{r} \int d \mathbf{r}^{\prime} \Psi_{n, s, \alpha}^{\dagger}(\mathbf{r}) \Psi_{n, s^{\prime}, \beta}^{\dagger}\left(\mathbf{r}^{\prime}\right) \\
& \times V\left(\mathbf{r}-\mathbf{r}^{\prime}\right) \Psi_{n, s^{\prime}, \beta}\left(\mathbf{r}^{\prime}\right) \Psi_{n, s, \alpha}(\mathbf{r})
\end{aligned}
$$

where the Coulomb potential $V(\mathbf{r})=e^{2} / \kappa r$ with $\kappa$ the dielectric constant of the substrate holding the graphene layer. The electron (spinor-)field operator is written as

$$
\Psi_{n, s, \alpha}(\mathbf{r})=\sum_{m}\langle\mathbf{r} \mid n, m, \alpha\rangle c_{s, \alpha, n, m}
$$

where $c_{s, \alpha, n, m}$ annihilates an electron of spin $s$ in valley $\alpha$, Landau level $n$, and orbital quantum number $m$. In Eq. (12), the terms that do not conserve the valley index are very small and have been neglected 17 .

To ensure the system's neutrality, an interaction between the C2DEG and a uniform positive background of density $n_{b}=N_{e} / S$ where $N_{e}$ is the number of electrons in level $n$ must be added to $V$. That interaction is given by

$$
V_{e-b}=-n_{b} N_{e} \int d \mathbf{r} V(\mathbf{r})
$$

where

$$
N_{e}=\sum_{\alpha, s} \int d \mathbf{r} \Psi_{n, s, \alpha}^{\dagger}(\mathbf{r}) \Psi_{n, s, \alpha}(\mathbf{r})
$$

Making the usual Hartree-Fock pairing of the field operators in Eq. (12), we get for the Hartree-Fock Hamiltonian

$$
\begin{aligned}
H_{H F}= & \sum_{s, \alpha, m} E_{s, n}^{0} c_{s, \alpha, n, m}^{\dagger} c_{s, \alpha, n, m} \\
& +\sum_{s, s^{\prime}} \sum_{\alpha, \beta} \sum_{m_{1} \ldots m_{4}} V_{m_{1}, m_{2}, m_{3}, m_{4}}^{n} \\
& \times\left\langle c_{s, \alpha, n, m_{1}}^{\dagger} c_{s, \alpha, n, m_{2}}\right\rangle c_{s^{\prime}, \beta, n, m_{3}}^{\dagger} c_{s^{\prime}, \beta, n, m_{4}} \\
& -\sum_{s, s^{\prime}} \sum_{\alpha, \beta} \sum_{m_{1} \ldots m_{4}} V_{m_{1}, m_{2}, m_{3}, m_{4}}^{n} \\
& \times\left\langle c_{s, \alpha, n, m_{1}}^{\dagger} c_{s^{\prime}, \beta, n, m_{4}}\right\rangle c_{s^{\prime}, \beta, n, m_{3}}^{\dagger} c_{s, \alpha, n, m_{2}} \\
& -\nu_{n} \sum_{s, \alpha, m_{1}, m_{2}} V_{m_{1}, m_{1}, m_{2}, m_{2}}^{n} c_{s, \alpha, n, m_{2}}^{\dagger} c_{s, \alpha, n, m_{2}},
\end{aligned}
$$

where the last term is the interaction with the positive background. 

by

The interactions $V_{m_{1}, m_{2}, m_{3}, m_{4}}^{n}$ in Eq. (16) are defined

$$
\begin{aligned}
& V_{m_{1}, m_{2}, m_{3}, m_{4}}^{n} \\
= & V_{m_{1}, m_{2}, m_{3}, m_{4}}^{0,0,0} \delta_{n, 0} \\
& +\frac{1}{4}\left[V_{m_{1}, m_{2}, m_{3}, m_{4}}^{|n|,|n|,|n|,|n|}+V_{m_{1}, m_{2}, m_{3}, m_{4}}^{|n|-|n|-1,|n|-1,|n|-1}\right] \Theta(|n|) \\
& +\frac{1}{4}\left[V_{m_{1}, m_{2}, m_{3}, m_{4}}^{|n|,|n|-|n|-1,|n|-1}+V_{m_{1}, m_{2}, m_{3}, m_{4}}^{|n|-1,|n|-1,|n|}\right] \Theta(|n|),
\end{aligned}
$$

where

$$
\begin{aligned}
V_{m_{1}, m_{2}, m_{3}, m_{4}}^{n_{1}, n_{2}, n_{3}, n_{4}}= & \int d \mathbf{r} \varphi_{n_{1}, m_{1}}^{*}(\mathbf{r}) \varphi_{n_{2}, m_{2}}(\mathbf{r}) \\
& \times \int d \mathbf{r}^{\prime} \frac{e^{2}}{\kappa\left|\mathbf{r}-\mathbf{r}^{\prime}\right|} \varphi_{n_{3}, m_{3}}^{*}\left(\mathbf{r}^{\prime}\right) \varphi_{n_{4}, m_{4}}\left(\mathbf{r}^{\prime}\right) .
\end{aligned}
$$

The matrix elements that are needed in Eq. (17) are all of the form $V_{m_{1}, m_{2}, m_{3}, m_{4}}^{n, n, q, q}$ and can be evaluated numerically using the following expression

$$
\begin{aligned}
& V_{m_{1}, m_{2}, m_{3}, m_{4}}^{n, n, q,} \\
= & \left(\frac{e^{2}}{\kappa \ell}\right) \sqrt{\frac{\operatorname{Min}\left(m_{1}, m_{2}\right) !}{\operatorname{Max}\left(m_{1}, m_{2}\right) !}} \sqrt{\frac{\operatorname{Min}\left(m_{3}, m_{4}\right) !}{\operatorname{Max}\left(m_{3}, m_{4}\right) !}} \\
& \times \delta_{m_{1}+m_{3}, m_{2}+m_{4}} \sqrt{2} \int_{0}^{\infty} d x e^{-2 x^{2}} x^{2\left|m_{1}-m_{2}\right|} \\
& \times L_{n}^{0}\left(x^{2}\right) L_{q}^{0}\left(x^{2}\right) L_{\operatorname{Min}\left(m_{1}, m_{2}\right)}^{\left|m_{1}-m_{2}\right|}\left(x^{2}\right) L_{\operatorname{Min}\left(m_{3}, m_{4}\right)}^{\left|m_{3}-m_{4}\right|}\left(x^{2}\right) .
\end{aligned}
$$

The Hamiltonian of Eq. (16) is very general and allows the calculation of skyrmion excitations with valley pseudospin texture, spin texture or even skyrmions with intertwined spin and valley pseudospin textures. In this paper, we restrict ourselves to situations where the quartet of state in Landau level $|n|>0$ is half-filled in which case the ground is spin polarized and spin-skyrmions excitations are possible and to $1 / 4$ or $3 / 4$ fillings in which cases the ground state is valley polarized and valley skyrmions are possible. Experiments show that the ground states in $n=0$ are more complex ${ }^{2}$ and we will not consider this Landau level. Indeed, for $\nu=0$, the ground state is probably not fully spin polarized and the nature of the broken-symmetry ground state is still debated 18 . At $\nu=-1$, experiments suggest that excitations contain both valley and spin flips.

At half filling, the states with up spins in both valleys are occupied. In a spin skyrmion excitation, an electron of spin $s=-1$ is added to the ground state and causes a certain number of spins $s=+1$ to flip to the $s=-1$ state in order to minimize the Coulomb exchange energy between electrons. These spins reversal can, in principle, occur in both valleys. But, because of the $\mathrm{SU}(2)$ valley symmetry of the Hamiltonian of Eq. (16), it is equivalent to consider that they originate from one of the valley only. When we do so, we assume that the other valley plays no role and can be considered as inert. When considering spin skyrmions only, we can thus restrict the Hilbert space in Landau level $n$ to one valley, say $\alpha=+1$, and to two spin orientations. In this way, we can work with a two- instead of a four-level system. The same principle can be applied to the ground state at $1 / 4$ or $3 / 4$ fillings. At $1 / 4$ filling, for example, state of up spins in valley $K_{+}$ (or any linear combination of $K_{+}$and $K_{-}$because of the $\mathrm{SU}(2)$ valley symmetry) are occupied. If we assume that spin flips are not possible because of the finite Zeeman coupling, than we have a two-level system with $K_{ \pm}$and up spins and excitations are valley skyrmions. At $3 / 4$ filling, we have a two-level system with states $K_{ \pm}$and down spins.

From now on, we drop the Landau level indices (except in the interaction $V^{n}$ ) and write the Hartree-Fock Hamiltonian as

$$
\begin{aligned}
H_{H F}= & \sum_{s, m} E_{s}^{0} \rho_{m, m}^{s, s} \\
& +\frac{1}{2} \sum_{s, s^{\prime}} \sum_{m_{1} \ldots m_{4}} V_{m_{1}, m_{2}, m_{3}, m_{4}}^{n}\left\langle\rho_{m_{1}, m_{2}}^{s, s}\right\rangle \rho_{m_{3}, m_{4}}^{s^{\prime},,^{\prime}} \\
& -\frac{1}{2} \sum_{s, s^{\prime}} \sum_{m_{1} \ldots m_{4}} V_{m_{1}, m_{2}, m_{3}, m_{4}}^{n}\left\langle\rho_{m_{1}, m_{4}}^{s, s^{\prime}}\right\rangle \rho_{m_{3}, m_{2}}^{s^{\prime}, s} \\
& -\nu_{n} \sum_{s, m_{1}, m_{2}} V_{m_{1}, m_{1}, m_{2}, m_{2}}^{n}\left\langle\rho_{m_{2}, m_{2}}^{s, s}\right\rangle \\
& +\frac{1}{2} \nu_{n}^{2} \sum_{m_{1}, m_{2}} V_{m_{1}, m_{1}, m_{2}, m_{2}}^{n},
\end{aligned}
$$

where we have defined the operator

$$
\rho_{m_{1}, m_{2}}^{s, s^{\prime}}=c_{s, m_{1}}^{\dagger} c_{s^{\prime}, m_{2}} .
$$

The last term in Eq. (20) is the background's electrostatic interaction $V_{b-b}=\frac{1}{2} n_{b}^{2} \int d \mathbf{r} \int d \mathbf{r}^{\prime} V\left(\mathbf{r}-\mathbf{r}^{\prime}\right)$ which must be included in $H_{H F}$ in order to correctly take into account the system's neutrality when computing excitation energies.

The Hamiltonian $H_{H F}$ contains the $s$ index and is written with spin skyrmions in mind. We give all the subsequent formulas for spin skyrmions. Valley skyrmions are easily treated by replacing $s$ with the valley index $\alpha$ in these formulas and neglecting the Zeeman term in the excitations energy.

We remark that, for $n=0$, the interactions $V_{m_{1}, m_{2}, m_{3}, m_{4}}^{n}$ given by Eq. (17) are identical to that of a conventional 2DEG. If one assumes a spin-polarized ground state for $n=0$ the quasiparticle and spinskyrmion excitation energies found from Eq. (20) are identical to those of a non-chiral 2DEG's which were computed in Refs. 4, 15 .

\section{QUASIPARTICLE AND SKYRMION EXCITATIONS}

In the two-level system, the ground state is given by 


$$
|G S\rangle=\prod_{m}^{\infty} c_{+, m}^{\dagger}|0\rangle
$$

which implies that

$$
\left\langle\rho_{m, m^{\prime}}^{s, s^{\prime}}\right\rangle=\delta_{m, m^{\prime}} \delta_{s, s^{\prime}} \delta_{s,+}
$$

Its energy is given by

$$
E_{G S}=-\frac{1}{2} N_{e} g \mu_{B} B-\frac{1}{2} \sum_{m_{1}, m_{2}} V_{m_{1}, m_{2}, m_{2}, m_{1}}^{n} .
$$

An quasi-electron excitation is obtained by adding one electron of spin $s=-1$ and angular momentum $m_{0}$ to the ground state i.e.

$$
|e\rangle=c_{-, m_{0}}^{\dagger}|G S\rangle
$$

and has

$$
\left\langle\rho_{m, m^{\prime}}^{s, s^{\prime}}\right\rangle=\left\{\begin{array}{cl}
\delta_{s, s^{\prime}} \delta_{m, m^{\prime}}, & \text { if } s=+1 \\
\delta_{m, m_{0}} \delta_{s, s^{\prime}} \delta_{m, m^{\prime}}, & \text { if } s=-1
\end{array}\right.
$$

The energy required to add one electron to the ground state is

$$
\Delta_{e}=\frac{1}{2} \Delta_{Z}
$$

and is independent of the value of $m_{0}$.

For the quasi-hole state,

$$
|h\rangle=c_{+, m_{0}}|G S\rangle
$$

with

$$
\left\langle\rho_{m, m^{\prime}}^{s, s^{\prime}}\right\rangle=\left\{\begin{array}{cc}
\delta_{s, s^{\prime}} \delta_{m, m^{\prime}}\left(1-\delta_{m, m_{0}}\right), & \text { if } s=+1 \\
0, & \text { if } s=-1
\end{array}\right.
$$

and the energy required to create this state is given by

$$
\Delta_{h}=\frac{1}{2} \Delta_{Z}+\sum_{m} V_{m, m_{0}, m_{0}, m}^{n}
$$

If follows that the energy required to create an HartreeFock electron-hole pair with both particles infinitely separated in space is given by

$$
\begin{aligned}
\Delta_{e-h} & =\Delta_{e}+\Delta_{h} \\
& =\Delta_{Z}+\sum_{m} V_{m, m_{0}, m_{0}, m}^{n}
\end{aligned}
$$

Numerically, the value of $\Delta_{e h}$ is independent of the choice of $m_{0}$. In fact

$$
\sum_{m} V_{m, m_{0}, m_{0}, m}^{n}=\left(\frac{e^{2}}{\kappa \ell}\right) \int_{0}^{\infty} \frac{d x}{2 \pi}\left|\Lambda_{n}(x)\right|^{2},
$$

with

$$
\begin{aligned}
\Lambda_{n}(x)= & \delta_{n, 0} e^{-x^{2} / 4}+\frac{1}{2} \Theta(|n|) e^{-x^{2} / 4} \\
& \times\left[L_{|n|}^{0}\left(\frac{x^{2}}{2}\right)+L_{|n|-1}^{0}\left(\frac{x^{2}}{2}\right)\right] .
\end{aligned}
$$

Following Ref. 15, the skyrmion state in Landau level $n$ is written as

$$
|S\rangle=\prod_{p=0}^{\infty}\left(u_{p} c_{+, p}^{\dagger}+v_{p} c_{-, p+1}^{\dagger}\right) c_{-, 0}^{\dagger}|0\rangle,
$$

with the constraint

$$
\left|u_{p}\right|^{2}+\left|v_{p}\right|^{2}=1
$$

This state has energy $E_{S}$. The quasiparticle state $|e\rangle=$ $c_{-, 0}^{\dagger}|G S\rangle$ corresponds to the limit $u_{p}=1$ and $v_{p}=0$ for all $p^{\prime} s$ i.e. to a zero-size skyrmion. The skyrmion excitation energy is given by

$$
\Delta_{S}=E_{S}-E_{G S}
$$

In the skyrmion state, one electron of spin down and quantum state $p=0$ is added to the C2DEG and, at the same time, the state with spin up and quantum number $p$ is combined with a state with spin down and quantum number $p+1$. The difference in angular momentum between the two states is $\Delta l_{z}=+\hbar$ and such pairing produces a $2 \pi$ counter-clockwise rotation of the spins in real space as shown in Fig. [5(a) below. It is easy to show that the state $|S\rangle$ describes a spin texture with a unit topological charge. The variational freedom in the wave function of this state allows deviations of the spin texture from that of the pure $N L \sigma$ model. Far from the origin, this state is locally identical to the ferromagnetic ground state and all spins point in the "up" direction. Near the origin, the projection of the total spin along the field direction becomes negative. The total increase (decrease) in the electron charge near the origin compared to the ferromagnetic ground state corresponds to one added electron(hole) for the skyrmion(antiskyrmion)

The total number of reversed spins in the skyrmion state is given by

$$
K=\sum_{p}\left|v_{p}\right|^{2}
$$

In a similar way, the antiskyrmion state is given by

$$
|a S\rangle=\prod_{p=1}^{\infty}\left(u_{p} c_{+, p}^{\dagger}+v_{p} c_{-, p+1}^{\dagger}\right)|0\rangle
$$

and the excitation energy for an antiskyrmion is

$$
\Delta_{a S}=E_{a S}-E_{G S}
$$

In this case, the difference in angular momentum is $\Delta l_{z}=$ $-\hbar$ and the rotation of the spins in real space is clockwise 
as shown in Fig. 5(b). The total number of reversed spin in the state $|a S\rangle$ is again given by Eq. (37). At a given value of the Zeeman coupling, $K$ is the same for skyrmion and antiskyrmion. In total, the number of down spins in a skyrmion-antiskyrmion pair is given by $2 K+1$ when counting the spin of the added electron and hole and $2 K$ gives the number of flipped spins. In an electron-hole excitation, $K=0$.

We remark that the skyrmion and antiskyrmion energies are modified by the filled levels that we have neglected but the energy to create an unbound skyrmionantiskyrmion pair $\Delta_{S-a S}=\Delta_{a S}+\Delta_{S}$ is not.

When the Zeeman coupling is zero, the excitation energy of a large-scale spin texture is given by the nonlinear sigma model (NL $\sigma \mathrm{M})$

$$
E_{N L \sigma M}=\frac{1}{2} \rho_{s} \int(\nabla \mathbf{m})^{2},
$$

where $|\mathbf{m}|=1$ is the spin field. In this $\mathrm{SU}(2)$-invariant limit, we know the exact spin stiffness which is given by

$$
\rho_{s}=\frac{1}{16 \pi} \frac{e^{2}}{\kappa \ell} \int_{0}^{\infty} d x x^{2} e^{-\frac{x^{2}}{2}}
$$

in Landau level $n=0$ and by

$$
\begin{aligned}
\rho_{s}= & \frac{1}{16 \pi} \frac{e^{2}}{\kappa \ell} \int d x x^{2} e^{-\frac{x^{2}}{2}} \\
& \times \frac{1}{4}\left[L_{|n|}\left(\frac{x^{2}}{2}\right)+L_{|n|-1}\left(\frac{x^{2}}{2}\right)\right]^{2}
\end{aligned}
$$

in other Landau levels. It follows that the exact energy of a single (large scale) skyrmion or antiskyrmion is given by $\stackrel{4,14}{ }$.

$$
E_{N L \sigma M}=4 \pi \rho_{s}
$$

Eq. (43) is the energy needed to create a neutral spin texture. The definition of this energy ${ }^{19}$ is different from the skyrmion excitation energy we introduced above. However, the energy to create an unbound S-aS pair is given by $\Delta_{N L \sigma M}=8 \pi \rho_{s}$ and this energy coincides ${ }^{20}$ with $\Delta_{S-a S}$ as given by Eqs. (36) and (39).

The S-aS excitation gaps $\Delta_{N L \sigma M}$ for different values of $n$ have been computed by Kun Yang et al. $\underline{6}$ for a $2 \mathrm{DEG}$ with Dirac bands and compared with the corresponding gaps for a $2 \mathrm{DEG}$ with parabolic bands. This comparison showed that $\Delta_{N L \sigma M}<\Delta_{e-h}$ for Landau levels $n=0,1,2,3$ so that the transport gap is dominated by S-aS pairs at these filling factors.

In the $N L \sigma$ model, the energy of a skyrmion is independent of its size and $K \rightarrow \infty$. When the Zeeman coupling is considered, skyrmions are smaller and it becomes necessary to consider the Hartree electrostatic energy as well. The Hartree energy favors large scale skyrmions while the Zeeman coupling favors small size skyrmions. The competition between these energies lead to an optimal size for the skyrmion at a given Zeeman coupling.
Fig. 8 shows the transports gaps $\Delta_{e-h}$ and $\Delta_{N L \sigma M}$ for several Landau levels at $\Delta_{Z}=0$. The transport gaps for the chiral and non-chiral 2DEG's are also listed in Table 1 of Ref. 6 .

\section{GREEN'S FUNCTION FORMALISM FOR SKYRMIONS}

In the skyrmion and antiskyrmion states, the only nonzero $\langle\rho\rangle^{\prime} s$ are given by

$$
\left\langle\rho_{p, p}^{s, s}\right\rangle,\left\langle\rho_{p \pm 1, p}^{-,+}\right\rangle,\left\langle\rho_{p, p \pm 1}^{+,-}\right\rangle \neq 0,
$$

where the upper(lower) sign in the subscripts is for skyrmion(antiskyrmion). To compute these average values, we define the matrix of Matsubara Green's functions

$$
G_{p}^{ \pm}(\tau)=\left(\begin{array}{cc}
G_{p, p}^{+,+}(\tau) & G_{p, p \pm 1}^{+,-}(\tau) \\
G_{p \pm 1, p}^{-,+}(\tau) & G_{p \pm 1, p \pm 1}^{-, \underline{1}}(\tau)
\end{array}\right)
$$

with

$$
G_{p, p^{\prime}}^{s, s^{\prime}}(\tau)=-\left\langle T_{\tau} c_{s, p}(\tau) c_{s^{\prime}, p^{\prime}}^{\dagger}(0)\right\rangle
$$

where $T_{\tau}$ is the imaginary time ordering operator. By definition, the $\langle\rho\rangle^{\prime} s$ are related to the Green's functions by the relation

$$
G_{p}^{ \pm}\left(\tau=0^{-}\right)=\left(\begin{array}{cc}
\left\langle\rho_{p, p}^{+,+}\right\rangle & \left\langle\rho_{p \pm 1, p}^{-,+}\right\rangle \\
\left\langle\rho_{p, p \pm 1}^{+,-}\right\rangle & \left\langle\rho_{p \pm 1, p \pm 1}^{-,-}\right\rangle
\end{array}\right) .
$$

Note that we must take $p=0,1,2, \ldots$ for a skyrmion and $p=1,2,3, \ldots$ for an antiskyrmion. To take into account the added electron or hole, we must in addition set $\left\langle\rho_{0,0}^{-,-}\right\rangle=1$ for the skyrmion and $\left\langle\rho_{0,0}^{+,+}\right\rangle=0$ for the antiskyrmion.

Using the Heisenberg equation of motion

$$
\hbar \frac{\partial}{\partial \tau}(\ldots)=[K,(\ldots)]
$$

with $K=H-\mu N_{e}$, where $\mu$ is the chemical potential, we get the following equation of motion for the Green's function $G_{p, p^{\prime}}^{s, s^{\prime}}(\tau)$ :

$$
\begin{aligned}
\hbar \frac{\partial}{\partial \tau} G_{p, p^{\prime}}^{s, s^{\prime}}(\tau)= & -\hbar \delta(\tau) \delta_{p, p^{\prime}} \delta_{s, s^{\prime}}-E_{s}^{0} G_{p, p^{\prime}}^{s, s^{\prime}}(\tau) \\
& -\sum_{s^{\prime \prime}} \sum_{m} V_{m, m, p, p}^{n}\left\langle\rho_{m, m}^{s^{\prime \prime}, s^{\prime \prime}}\right\rangle G_{p, p^{\prime}}^{s, s^{\prime}}(\tau) \\
& +\sum_{m} V_{m, p, p, m}^{n}\left\langle\rho_{m, m}^{s, s}\right\rangle G_{p, p^{\prime}}^{s, s^{\prime}}(\tau) \\
& +\sum_{m} V_{m \pm 1, p \pm 1, p, m}^{n}\left\langle\rho_{m \pm 1, m}^{-,+}\right\rangle G_{p \pm 1, p^{\prime}}^{-, s^{\prime}}(\tau) \delta_{s,+} \\
& +\sum_{m} V_{m, p \mp 1, p, m \pm 1}^{n}\left\langle\rho_{m, m+1}^{+,-}\right\rangle G_{p \mp 1, p^{\prime}}^{+, s^{\prime}}(\tau) \delta_{s,-} \\
& +\sum_{m} V_{m, m, p, p}^{n} G_{p, p^{\prime}}^{s, s^{\prime}}(\tau)
\end{aligned}
$$


This equation can be written in an obvious matrix form as

$$
\left[\left(i \omega_{n}+\mu\right) I-\frac{1}{\hbar} F_{p}^{ \pm}\right] G_{p}^{ \pm}\left(i \omega_{n}\right)=I,
$$

where $I$ is the $2 \times 2$ units matrix. The components of the $2 \times 2$ matrices $F_{p}^{ \pm}$are given by (with $\left\langle\rho_{m, m}\right\rangle \equiv$ $\left.\sum_{s}\left\langle\rho_{m, m}^{s, s}\right\rangle\right)$

$$
\begin{aligned}
\left(F_{p}^{ \pm}\right)_{1,1}= & E_{+}^{0}+\sum_{m} V_{m, m, p, p}^{n}\left[\left\langle\rho_{m, m}\right\rangle-1\right] \\
& -\sum_{m} V_{m, p, p, m}^{n}\left\langle\rho_{m, m}^{+,+}\right\rangle \\
\left(F_{p}^{ \pm}\right)_{2,2}= & E_{-}^{0}+\sum_{m} V_{m, m, p \pm 1, p \pm 1}^{n}\left[\left\langle\rho_{m, m}\right\rangle-1\right] \\
& -\sum_{m} V_{m, p \pm 1, p \pm 1, m}^{n}\left\langle\rho_{m, m}^{-,-}\right\rangle \\
\left(F_{p}^{ \pm}\right)_{1,2}= & -\sum_{m} V_{m \pm 1, p \pm 1, p, m}^{n}\left\langle\rho_{m \pm 1, m}^{-,+}\right\rangle \\
\left(F_{p}^{ \pm}\right)_{2,1}= & -\sum_{m} V_{m, p, p \pm 1, m \pm 1}^{n}\left\langle\rho_{m, m \pm 1}^{+,-}\right\rangle
\end{aligned}
$$

where the summations extend over all values of $m$ in the diagonal elements of $F_{p}^{ \pm}$and from $m=0(m=1)$ to infinity for skyrmions (antiskyrmions) in the off-diagonal elements.

The matrices $F^{ \pm}$are Hermitian and can be diagonalized by a unitary transformation

$$
F_{p}^{ \pm}=U_{p}^{ \pm} D_{p}^{ \pm}\left(U_{p}^{ \pm}\right)^{\dagger}
$$

with $D_{p}^{ \pm}$the diagonal matrix of the eigenvalues $\left(d_{p}^{ \pm}\right)_{k}$ of $F_{p}^{ \pm}$. If follows that

$$
\left[G_{p}^{ \pm}\left(i \omega_{n}\right)\right]_{i, j}=\sum_{k} \frac{\left(U_{p}^{ \pm}\right)_{i, k}\left[\left(U_{p}^{ \pm}\right)\right]_{k, j}^{\dagger}}{i \omega_{n}+\mu-\left(d_{p}^{ \pm}\right)_{k}}
$$

and

$$
\left[G_{p}^{ \pm}\left(\tau=0^{-}\right)\right]_{i, j}=\left(U_{p}^{ \pm}\right)_{i, n}\left[\left(U_{p}^{ \pm}\right)^{\dagger}\right]_{n, j},
$$

with $n=1$ when $\left(d_{p}^{ \pm}\right)_{1}<\left(d_{p}^{ \pm}\right)_{2}$ and $n=2$ otherwise.

The Hartree-Fock self-consistent Eq. (50) is solved numerically using an iterative scheme until self-consistency is achieved for the $\langle\rho\rangle^{\prime} s$. The excitation energy for skyrmion (upper sign) and antiskyrmion (lower sign) are then computed using (the Zeeman term is absent for valley skyrmions):

$$
\begin{aligned}
\Delta_{S / a S}= & \left.H_{ \pm}\right\rangle-E_{G S} \\
= & -\frac{1}{2} g \mu_{B} B \sum_{s, m} s\left[\left\langle\rho_{m, m}^{s, s}\right\rangle-\delta_{s,+}\right] \\
& +\frac{1}{2} \sum_{m, p} V_{m, m, p, p}^{n}\left[\left(\left\langle\rho_{m, m}\right\rangle-2\right)\left\langle\rho_{p, p}\right\rangle+1\right] \\
& -\frac{1}{2} \sum_{s} \sum_{m, p} V_{m, p, p, m}^{n}\left[\left\langle\rho_{m, m}^{s, s}\right\rangle\left\langle\rho_{p, p}^{s, s}\right\rangle-\delta_{s,+}\right] \\
& -\frac{1}{2} \sum_{m, p} V_{m, p, p \pm 1, m \pm 1}^{n}\left\langle\rho_{m, m \pm 1}^{+,-}\right\rangle\left\langle\rho_{p \pm 1, p}^{-,+}\right\rangle \\
& -\frac{1}{2} \sum_{m, p} V_{m \pm 1, p \pm 1, p, m}^{n}\left\langle\rho_{m \pm 1, m}^{-,+}\right\rangle\left\langle\rho_{p, p \pm 1}^{+,-}\right\rangle
\end{aligned}
$$

The excitation energy combines the Zeeman cost of the flipped spins, the Coulomb self-interaction of the excess charge and the exchange energy cost associated the rotation of the spins with respect to the ferromagnetic ground state.

The change in the electronic density and spin pattern in real space can easily be obtained from the $\langle\rho\rangle^{\prime} s$ by using

$$
\delta n(\mathbf{r})=\sum_{m} \Lambda_{m}^{n}(\mathbf{r})\left[\left\langle\rho_{m, m}^{+,+}\right\rangle+\left\langle\rho_{m, m}^{-,-}\right\rangle-1\right],
$$

and

$$
\begin{aligned}
\delta S_{x}(\mathbf{r}) & =\frac{\hbar}{2} \sum_{m}\left[\Upsilon_{m, \pm}^{n}(\mathbf{r})\left\langle\rho_{m, m \pm 1}^{+,-}\right\rangle+c . c .\right] \\
\delta S_{y}(\mathbf{r}) & =\frac{\hbar}{2 i} \sum_{m}\left[\Upsilon_{m, \pm}^{n}(\mathbf{r})\left\langle\rho_{m, m \pm 1}^{+,-}\right\rangle-c . c .\right] \\
\delta S_{z}(\mathbf{r}) & =\frac{\hbar}{2} \sum_{m} \Lambda_{m}^{n}(\mathbf{r})\left[\left\langle\rho_{m, m}^{+,+}\right\rangle-\left\langle\rho_{m, m}^{-,-}\right\rangle-1\right]
\end{aligned}
$$

with the definitions

$$
\begin{aligned}
\Lambda_{m}^{0}(\mathbf{r}) & =\left|\varphi_{0, m}(\mathbf{r})\right|^{2}, \\
\Upsilon_{m, \pm}^{0}(\mathbf{r}) & =\varphi_{0, m}^{*}(\mathbf{r}) \varphi_{0, m \pm 1}(\mathbf{r}),
\end{aligned}
$$

and

$$
\begin{aligned}
\Lambda_{m}^{|n|>0}(\mathbf{r})= & \frac{1}{2}\left[\left|\varphi_{|n|, m}(\mathbf{r})\right|^{2}+\left|\varphi_{|n|-1, m}(\mathbf{r})\right|^{2}\right], \\
\Upsilon_{m, \pm}^{|n|>0}(\mathbf{r})= & \frac{1}{2} \varphi_{|n|, m}^{*}(\mathbf{r}) \varphi_{|n|, m \pm 1}(\mathbf{r}) \\
& +\frac{1}{2} \varphi_{|n|-1, m}^{*}(\mathbf{r}) \varphi_{|n|-1, m \pm 1}(\mathbf{r}) .
\end{aligned}
$$

Again, the summations over $m$ in $\delta S_{x}$ and $\delta S_{y}$ run from $m=0(m=1)$ to infinity for skyrmions(antiskyrmions).

\section{NUMERICAL RESULTS FOR THE EXCITATION ENERGIES}

The Green's function approach just described is wellsuited to compute the energy of a finite-size skyrmion 
but there is a practical difficulty with it. In practice we are forced to truncate the set of single-particle angular momenta that we include in the description of both the ferromagnetic ground state and the charged excitations at a finite value $m_{\max }$. Since the single-particle orbital with angular momentum $m$ is localized near a ring with radius $\sqrt{(2 m+1)} \ell$, this is equivalent to working with a finite-size electron disk of radius $R \approx \sqrt{2 m_{\max }} \ell$. The skyrmion excitation energy $\Delta_{S}$ will be given accurately by our method if the tail of the disturbance associated with the charged excitation does not extend to the edge of the disk. When the Zeeman coupling $\widetilde{g} \equiv \Delta_{Z} /\left(e^{2} / \kappa \ell\right)$ decreases below a certain value, the skyrmion size becomes large and this condition is not satisfied.

The Landau level wave functions obey the identity

$$
\sum_{m=0}^{m_{\max }=\infty}\left|\varphi_{n, m}(\mathbf{r})\right|^{2}=\frac{1}{2 \pi \ell^{2}} .
$$

If $m_{\max }=160$, Eq. (67) is satisfied numerically for $r_{\max } / \ell \lesssim 15$ while for $m_{\max }=1000$, it is satisfied for $r / \ell \lesssim 35$. In our numerical calculations, we set $m_{\max }=1000$. It follows that the charged excitation that we compute must be well contained in a disk a radius $r_{\max } / \ell \lesssim 35$ for our calculation to be reliable.

Fig. 11 shows the energy $E_{S}$ of one skyrmion and the corresponding number of down spins $N_{\downarrow}=2 K+1$ as a function of the Zeeman coupling $\widetilde{g}$ for different values of the maximum angular momentum $m_{\max }$ up to 2000 (In all our numerical calculations, we use $\kappa=2.5$ for the dielectric constant of the substrate.) A good convergence of $E_{S}$ and $N_{\downarrow}$ is obtained for $\widetilde{g} \gtrsim 0.001$ with $m_{\max }=$ 1000. Due to the variational nature of the Hartree-Fock calculation, the energies approach their asymptotic value much more rapidly with increasing $m_{\max }$ than estimates of the optimal value of $N_{\downarrow}$. The values of $\Delta_{S-a S}$ at small Zeeman coupling are thus more reliable than those of $N_{\downarrow}$.

Fig. 2] shows the behavior of the gaps $\Delta_{e-h}$ and $\Delta_{S-a S}$ with Zeeman coupling $\widetilde{g}$ for Landau levels $n=1,2,3$. The value of the gap $\Delta_{N L \sigma M}$ is also indicated for each Landau level. The upward vertical arrows are placed at values of $\widetilde{g}$ corresponding to the total magnetic fields $B=$ $15,25,30 \mathrm{~T}$ with $B_{\perp}=15 \mathrm{~T}$. This allows a comparison of our results with Fig. 2(e) of Ref. 2 where the activation gap measured in a tilted-field experiment is plotted as a function of the total magnetic field for $n=-1,-2$. (Note that the theoretical activation energies depend only on $|n|$.) In such experiments, the magnetic length is actually defined by $\ell=\sqrt{\hbar c / e B_{\perp}}$ and $B_{\perp}$ is kept fixed while the magnetic field is tilted. This is equivalent to changing $\widetilde{g}$ and keeping the filling factor fixed.

In Fig. 2, the gap $\Delta_{e-h}$ decreases with increasing Landau level index reflecting the decrease of the exchange energy with $n$ in Eq. (31). On the contrary, $\Delta_{N L \sigma M}$ and $\Delta_{S-a S}$ both increase with $n$ in the small Zeeman range where skyrmions exist for $n=1$ and $n=2$ (the region near the down arrow in the figure). If the $n=2$ skyrmion were to persist to larger values of $\widetilde{g}$, the $\Delta_{S-a S}$

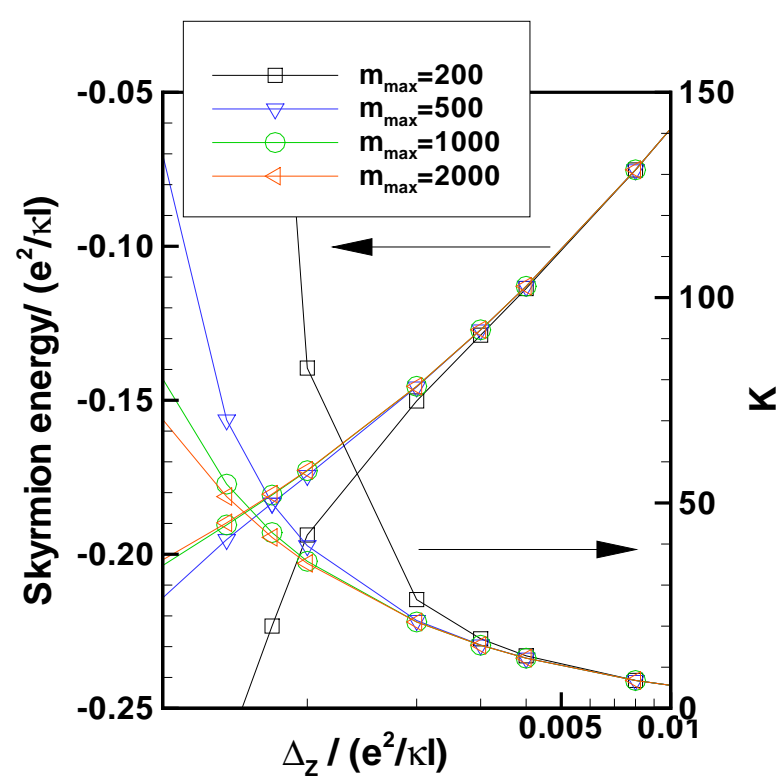

FIG. 1: (Color online) Energy of one skyrmion $E_{S}$ and the corresponding number of down spins $N_{\downarrow}=2 K+1$ as a function of the Zeeman coupling for different values of the maximum angular momentum used in the computation.

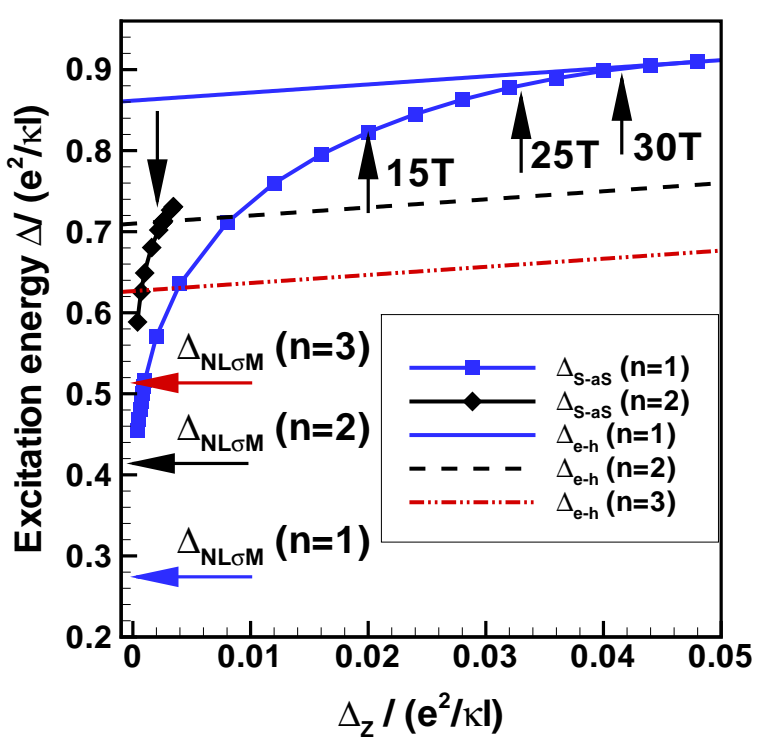

FIG. 2: (Color online) Excitation energy of a skyrmionantiskyrmion pair $\Delta_{S K}-A S K$ and an electron-hole pair $\Delta_{e-h}$ as a function of the Zeeman coupling $\Delta_{Z} /\left(e^{2} / \kappa \ell\right)$ for Landau levels $n=1,2,3$. The horizontal arrows indicate the value of the skyrmion-antiskyrmion gap $\Delta_{N L \sigma M}$ calculated in the non-linear $\sigma$ model. The upward vertical arrows are positioned at the value of $\widetilde{g}$ corresponding to total magnetic fields $B=15,25,30 \mathrm{~T}$ when $B_{\perp}=15 \mathrm{~T}$. The downward arrow points to the value of $\widetilde{g}_{c}$ for $n=2$. 
gap would actually decrease with $n$ at large $\widetilde{g}$ but this does not happen in our calculation.

The maximal value, $\widetilde{g}_{c}$, of the Zeeman coupling for which $\Delta_{S-a S}<\Delta_{e-h}$ decreases dramatically with Landau level level index as shown in Fig. 2. For example, the value of $\widetilde{g}_{c} \approx 0.0026$ for $n=2$ is one order of magnitude lower than that for $n=1$. This makes skyrmions difficult to calculate in higher Landau levels. Interestingly, we find that for $n=2, \Delta_{S-a S}$ goes over $\Delta_{e-h}$ for $g \geq \widetilde{g}_{c}$ $\left(\widetilde{g}_{c}\right.$ is indicated by a downward arrow in Fig. 2) instead of reaching $\Delta_{e-h}$ smoothly as is the case for $n=1$. At the crossing point $\widetilde{g}_{c}$ for $n=2$, the number of down spins, $N_{\downarrow}$ in the S-aS pair (see Fig. 3) is $\approx 25$, a large value. A similar jump in the number of down spins at the transition from S-aS pair to electron-hole pair was predicted theoretically for skyrmions in a conventional 2DEG when the finite width of the well was taken into account and at filling factor $\nu=3 \stackrel{8}{\text {. }}$. This jumps suggests that the spin polarization of the C2DEG could change abruptly at $\widetilde{g}_{c}$. This first order transition has been seen experimentally in a conventional 2DEG at $\nu=1^{21}$ and also in a conventional bilayer 2DEG at $\nu=1$ when the electrons occupy only one of the two layers ${ }^{22}$. Our calculation shows that it can also happen in graphene.

The number of down spins $N_{\downarrow}>1$ for a S-aS pair while $N_{\downarrow}=1$ for an electron hole pair. Fig. 3 shows that the rapid increase in energy of $\Delta_{S-a S}$ with $\widetilde{g}$ is associated with a rapid decrease in $N_{\downarrow}$. The number of down spins varies roughly linearly with $\widetilde{g}$ in between $B_{\perp}=15 \mathrm{~T}$ and $B_{\perp}=30 \mathrm{~T}$ but not at smaller values of the Zeeman coupling. At $B_{\perp}=15 \mathrm{~T}, N_{\downarrow} \approx 6$ for $n=1$ corresponding to $K=2.5$ reversed spins per skyrmion. Fig. 3 shows that, for the same Zeeman coupling, $K$ is smaller for a $n=2$ than for a $n=1$ skyrmion.

Fig. 4 shows $\delta n(r)$, the change in the density of the C2DEG with respect to the ferromagnetic ground state density $n_{G S}(r)=1 / 2 \pi \ell^{2}$ when a skyrmion is added to the ground state. (Because of the electron-hole symmetry of the Hamiltonian near half-filling, $\delta n_{S}(r)=-\delta n_{a S}(r)$ ). We can define the size or radius of a skyrmion, $r_{s k y}$, by

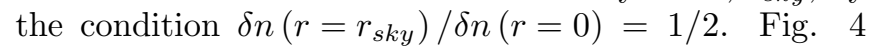
shows that the size of the skyrmions shrinks with increasing Zeeman coupling and also with increasing Landau level index at fixed Zeeman coupling. For $\widetilde{g}=0.002$, the skyrmion size for $n=1$ is $r_{s k y} / \ell \approx 2$ and the tail of the $\delta n(r)$ is well within the maximal radius $r_{\max } / \ell=35$ discussed above.

For $n=3$, the crossing point $\widetilde{g}_{c}$ occurs at a value of $\widetilde{g} \lesssim 0.0002$ where $N_{\downarrow}$ is very large, suggesting that the skyrmion size at that Zeeman coupling is already beyond the limit of reliability of our approach. Since the $\mathrm{NL} \sigma \mathrm{M}$ result indicates that skyrmions are the lowestenergy charged excitations for $n=3$, we can conclude that, if they persist to finite Zeeman coupling, it is certainly in a very narrow range of $\widetilde{g}$, approximately an order of magnitude smaller than for $n=2$.

The spin texture $\mathbf{S}_{\|}(\mathbf{r})$ for a skyrmion and an antiskyrmion excitations in $n=1$ at $\widetilde{g}=0.011$ is plotted in Fig. 5 with the component $S_{z}(\mathbf{r})$ given by the superimposed density plot. The in-plane component of the spin makes a $2 \pi$ counterclockwise (skyrmion) or clockwise (antiskyrmion) rotation around the center of the topological charge.

Finally, the magnitude of the gap $\Delta_{S-a S} \approx e^{2} / \kappa \ell$. For $B_{\perp}=15 \mathrm{~T}, e^{2} / \kappa \ell=1011 \mathrm{~K}$ and so $\Delta_{S-a S} \approx 910 \mathrm{~K}$ at $B=30 \mathrm{~T}$ for $n=1$.

Our results can be compared with those of Ref. 2 (see Fig. 2(e) of this paper) where the transport gap was measured at total magnetic fields $B=15,25,30 \mathrm{~T}$ with the perpendicular magnetic field $B_{\perp}=15 \mathrm{~T}$ kept fixed. In this experiment, the electronic density was varied in order to study the spin-texture excitations at filling factors $\nu=-4,-8,-12$ corresponding to half-filling of Landau levels $n=-1,-2,-3$. At $B=15,25,30 \mathrm{~T}$, according to our calculations, the transport gap is given by $\Delta_{S-a S}^{(n=1)}$ in $n=1$ and by $\Delta_{e-h}^{(n=2)}, \Delta_{e-h}^{(n=3)}$ in $n=2$ and $n=3$ with the ordering $\Delta_{S-a S}^{(n=1)}>\Delta_{e-h}^{(n=2)}>\Delta_{e-h}^{(n=3)}$. This ordering is consistent with the experimental result except for $n=2$. In this case, the experiment measures a small number of spin flips i.e. $N_{\downarrow} \approx 1.4$ suggesting that $\Delta_{S-a S}^{(n=2)}<\Delta_{e-h}^{(n=2)}$. We cannot explain this difference with our model of skyrmion excitations.

Another difference between the experimental and theoretical results is the size of the transport gap. For example, the experimental value of $\Delta_{S-a S}^{(n=1)} \approx 75 \mathrm{~K}$ at $B=30$ $\mathrm{T}$ while we find $\Delta_{S-a S} \approx 910 \mathrm{~K}$, a much larger value. Several effects may affect our results such as disorder, Landau level mixing ${ }^{7}$ and screening. In a conventional 2DEG, taking into account the quantum well width ${ }^{\underline{8}}$ is known to decrease the excitation energy but this effect is not present in graphene. In the remainder of this paper, we study the corrections due to screening since they are easy to include in our calculation and they lead to a substantial decrease of the gap. We leave disorder and Landau mixing effects to further work. Valley skyrmions were also studied at filling factors $\nu=-3,-5$ in Ref. 2 . We comment on them in the next section.

In closing this section, we remark that we have shown and commented here our results for the excitation energy of a skyrmion-antiskyrmion pair. Nevertheless, we have verified that whenever this energy is smaller than the corresponding electron-hole pair energy, the skyrmion (antiskyrmion) energy is smaller than the electron(hole) energy.

\section{SCREENING CORRECTIONS TO THE EXCITATION GAPS}

To include screening, we follow the approach of Ref. 23 where it was shown that when the Landau levels other than the partially filled level are integrated out, the low-frequency dynamics of the 2DEG is described by the electrons belonging to the partially filled Landau level but the interaction between these electrons (and 


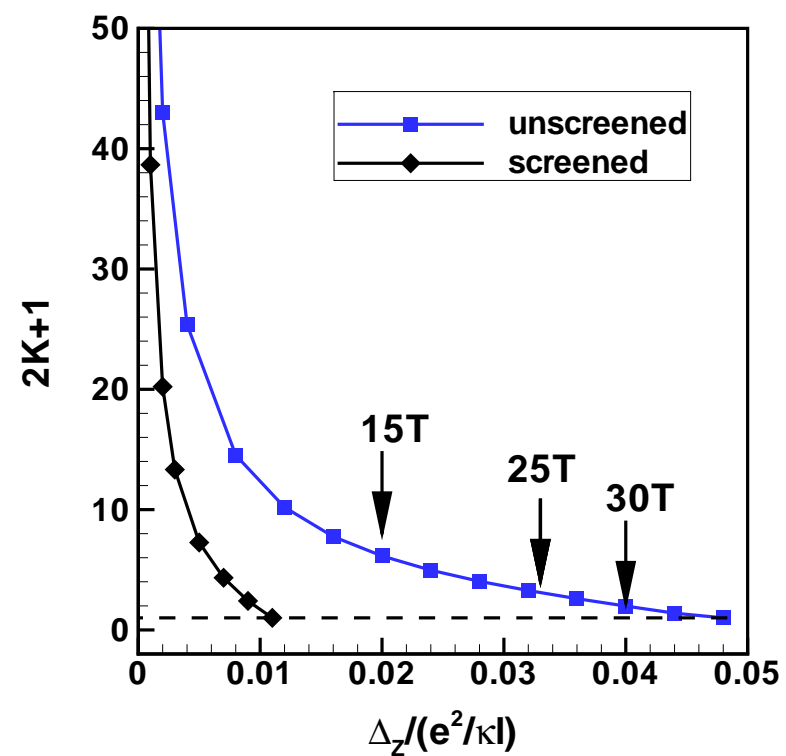

FIG. 3: (Color online) Number of down spins $N_{\downarrow}=2 K+1$ in a skyrmion-antiskyrmion pair as a function of the Zeeman coupling $\Delta_{Z} /\left(e^{2} / \kappa \ell\right)$ for Landau level $n=1$ with and without screening corrections. The arrows are placed that the values of $\widetilde{g}$ where the total magnetic fields $B=15,25,30 \mathrm{~T}$ when the perpendicular component $B_{\perp}=15 \mathrm{~T}$. The dashed line indicates $N_{\downarrow}=1$, the electron-hole limit.

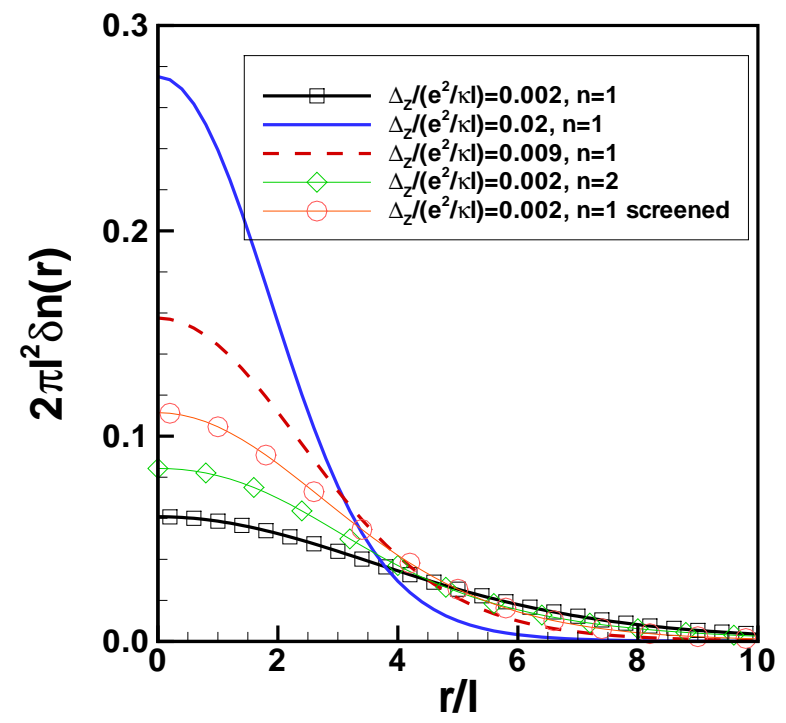

FIG. 4: (Color online)Profile of the induced density $\delta n(r)$ when a skyrmion is added to the ground state in Landau level $n=1$ for several values of the Zeeman coupling $\Delta_{Z} /\left(e^{2} / \kappa \ell\right)$. The profiles for the screened skyrmion in $n=1$ and the unscreened skyrmion in $n=2$ are also shown.
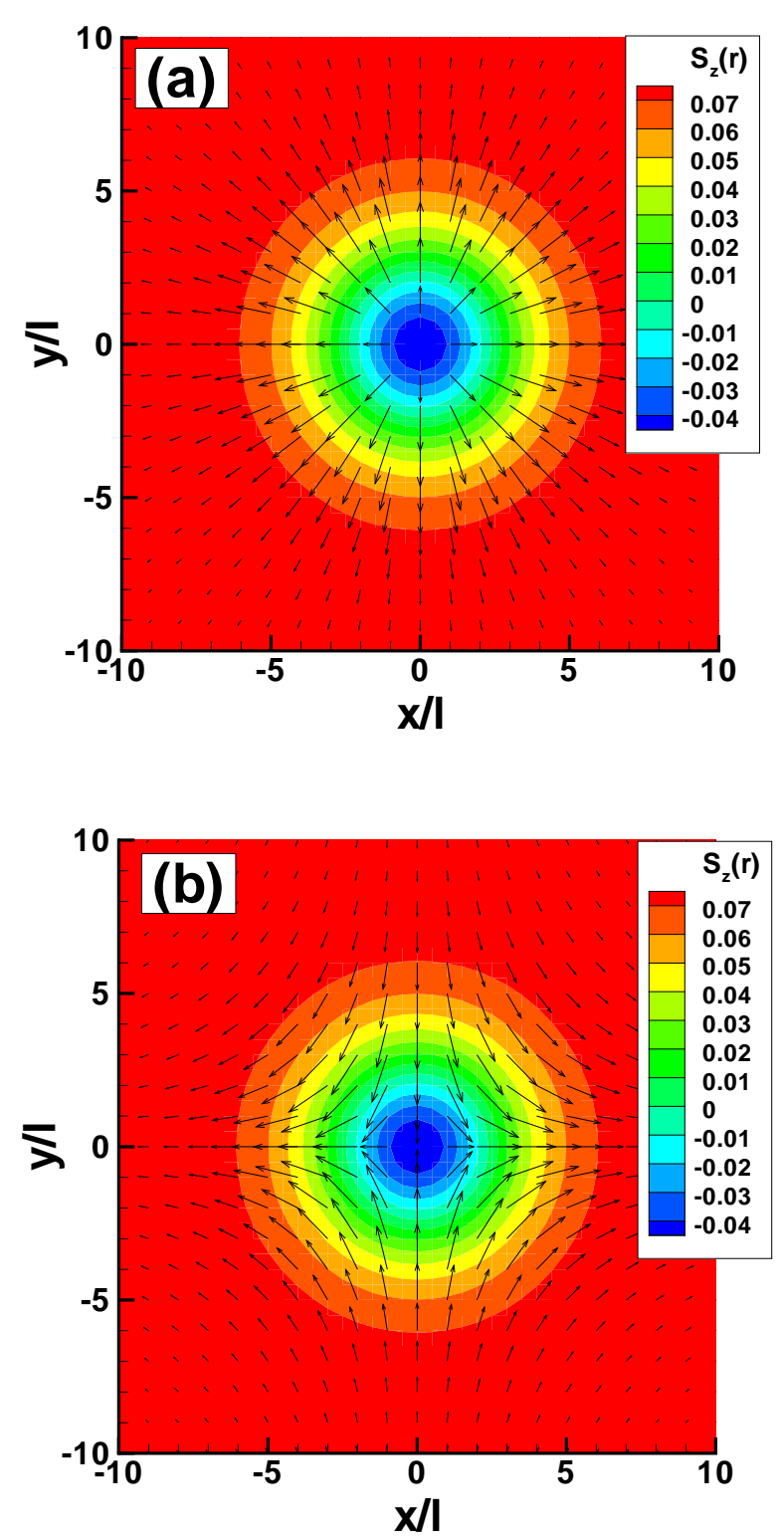

FIG. 5: (Color online)Spin texture in the $x-y$ plane for a (a) skyrmion and (b) an antiskyrmion in Landau level $n=1$ at Zeeman coupling $\Delta_{Z} /\left(e^{2} / \kappa \ell\right)=0.011$. The density plot shows $S_{z}(r)$ in units of $\hbar /\left(2 \pi \ell^{2}\right)$.

with the positive charge of the background) is renormalized due to the polarizability of all the other Landau levels. In the Hartree-Fock approximation, this renormalization amounts to screen both the Hartree and Fock interactions ${ }^{24}$. The bare Coulomb interaction $V(q)=$ $2 \pi e^{2} / \kappa q$ must then be replaced by $V(q)=2 \pi e^{2} / \varepsilon(q) \kappa q$ where $\varepsilon(q)$ is the static dielectric function calculated in the random-phase approximation (RPA). Such procedure was used, for example, in the study of inhomogeneous states such as bubble and stripe phases in quantum Hall systems $\frac{25}{}$. 
We follow this procedure by using the screened Coulomb interaction in the matrix elements of the Coulomb interaction in Eq. (18). The matrix elements are recalculated by inserting the dielectric function i.e. $\left(\frac{e^{2}}{\kappa \ell}\right) \int_{0}^{\infty} d x e^{-2 x^{2}}(\ldots) \rightarrow\left(\frac{e^{2}}{\kappa \ell}\right) \int_{0}^{\infty} d x \frac{e^{-2 x^{2}}}{\varepsilon(x)}(\ldots)$ where $x=q \ell / \sqrt{2}$ in Eq. (19). The dielectric function is evaluated in the random-phase approximation and is given by

$$
\varepsilon(\mathbf{q})=1-\frac{2 \pi e^{2}}{q} \chi^{0, R}(\mathbf{q}, \omega=0),
$$

where $\chi^{0, R}(\mathbf{q}, \omega)$ is the retarded density response function computed for a non-interacting C2DEG in a magnetic field. More precisely,

$$
\begin{aligned}
\varepsilon(\mathbf{q})= & 1+\frac{e^{2} / \kappa \ell}{\hbar \omega_{c}^{*}} \frac{1}{q \ell} \sum_{\alpha, s} \sum_{n, n^{\prime}}\left|\Xi_{n, n^{\prime}}(\mathbf{q})\right|^{2} \\
& \times \frac{\nu_{n, \alpha, s}-\nu_{n^{\prime}, \alpha, s}}{\operatorname{sgn}\left(n^{\prime}\right) \sqrt{\left|n^{\prime}\right|}-\operatorname{sgn}(n) \sqrt{|n|}},
\end{aligned}
$$

where $\nu_{n, \alpha, s}$ is the filling factor of Landau level $n$ with valley index $\alpha$ and spin $s$ and the function

$$
\begin{aligned}
\Xi_{n, n^{\prime}}(\mathbf{q})= & \frac{1}{2} \Theta(|n|) \Theta\left(\left|n^{\prime}\right|\right) \\
& \times\left[F_{|n|,\left|n^{\prime}\right|}(\mathbf{q})+\operatorname{sgn}(n) \operatorname{sgn}\left(n^{\prime}\right) F_{|n|-1,\left|n^{\prime}\right|-1}\right. \\
& +\frac{1}{\sqrt{2}}\left[\delta_{n, 0} \Theta\left(\left|n^{\prime}\right|\right)+\delta_{n^{\prime}, 0} \Theta(|n|)\right] F_{|n|,\left|n^{\prime}\right|}(\mathbf{q}) \\
& +\delta_{n, 0} \delta_{n^{\prime}, 0} F_{0,0}(\mathbf{q})
\end{aligned}
$$

with

$$
\begin{aligned}
F_{n, n^{\prime}}(\mathbf{q})= & \left(\frac{n^{\prime} !}{n !}\right)^{1 / 2}\left[\frac{\left(q_{y}+i q_{x}\right) \ell}{\sqrt{2}}\right]^{n-n^{\prime}} \\
& \times L_{n^{\prime}}^{n-n^{\prime}}\left(\frac{q^{2} \ell^{2}}{2}\right) e^{-q^{2} \ell^{\ell 2} / 4}
\end{aligned}
$$

for $n \geq n^{\prime}$. For $n<n^{\prime} F_{n, n^{\prime}}(\mathbf{q})=\left[F_{n^{\prime}, n}(-\mathbf{q})\right]^{*}$. We have defined the effective cyclotron energy $\hbar \omega_{c}^{*}=\sqrt{2} \hbar v_{F} / \ell$ so that

$$
\frac{e^{2} / \kappa \ell}{\hbar \omega_{c}^{*}}=\frac{1}{\sqrt{2} \kappa} \alpha^{*}
$$

where $\alpha^{*}=e^{2} / \hbar v_{F}$ is the effective fine structure constant for graphene. The dielectric function at integer filling $\nu$ of electrons is equal to the dielectric function at integer filling $\nu$ of holes. We write this as $\varepsilon_{\nu}=\varepsilon_{-\nu}$.

The dielectric function $\varepsilon(\mathbf{q})$ of the C2DEG has been evaluated previously ${ }^{26}$. We show our results obtained at different filling factors in Fig. 6. In our calculation we include Landau levels $m \in[-800,800]$ in the summations in Eq. (69). The dielectric function $\varepsilon(q)=1$ at $q=0$ and $q \rightarrow \infty$. It is maximal around $q \ell \approx 1$ and increases with increasing filling factors. In particular, screening is

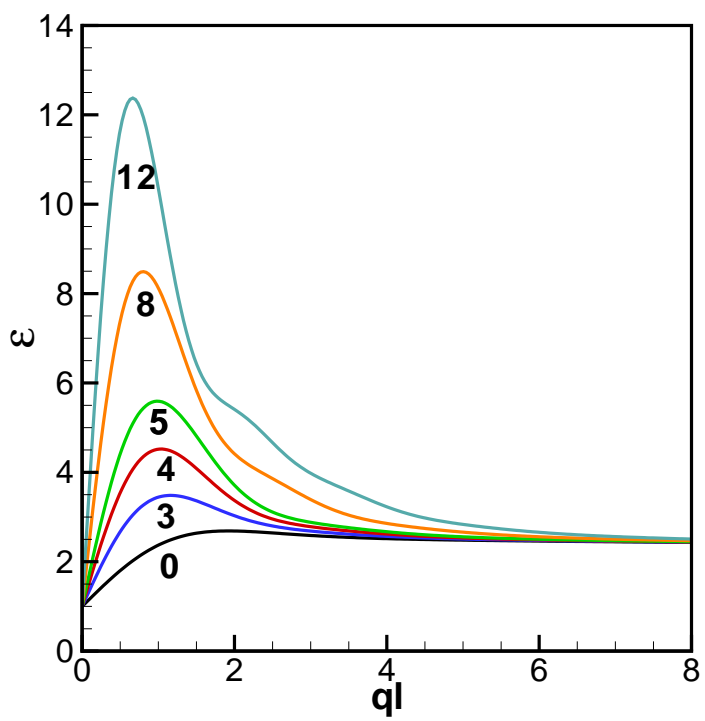

FIG. 6: (Color online)Static dielectric function computed in the random-phase approximation at different filling factors $|\nu|$ (indicated by the number below each curve) in Landau levels $|n|=0,1,2,3$.

larger at 3/4 filling of a given Landau level than at 1/4 (q) fllling as shown in Fig. 6 for $|n|=2$.

In a conventional 2DEG, the dielectric function is given by

$\varepsilon(\mathbf{q})=1+2\left(\frac{e^{2} / \kappa \ell}{\hbar \omega_{c}}\right) \frac{1}{q \ell} \sum_{s} \sum_{n, n^{\prime}}\left|F_{n, n^{\prime}}(\mathbf{q})\right|^{2} \frac{\nu_{n, s}-\nu_{n^{\prime}, s}}{n^{\prime}-n}$

where $n, n^{\prime}=0,1,2, \ldots$ Since $\left(e^{2} / \kappa \ell\right) / \hbar \omega_{c} \sim 1 / \sqrt{B}$, screening is less important at large magnetic fields in a conventional 2DEG than in graphene.

We have recomputed the energy of a Hartree-Fock electron-hole pair, $\Delta_{e-h}^{(S)}$, a S-aS pair, $\Delta_{S-a S}^{(S)}$, as well as the $N L \sigma$ model result $\Delta_{N L \sigma M}^{(S)}$ using the screened matrix elements. We use the superscript $(S)$ indicates the screened gaps. The gaps $\Delta_{e-h}^{(S)}$ and $\Delta_{N L \sigma M}^{(S)}$ are given by

$$
\Delta_{e-h}^{(S)}=g \mu_{B} B+\left(\frac{e^{2}}{\kappa \ell}\right) \int_{0}^{\infty} \frac{d x}{2 \pi} \frac{\left|\Lambda_{n}(x)\right|^{2}}{\varepsilon(x)},
$$

(with $\Lambda_{n}(x)$ defined in Eq. (33)) and

$$
\Delta_{N L \sigma M}^{(S)}=8 \pi \rho_{s}^{(S)},
$$

with $\int d x x^{2}(\ldots)$ replaced by $\int d x \frac{x^{2}}{\varepsilon(x)}(\ldots)$ in the definition of the spin stiffness in Eqs. (41) and (42). We remark that, as in the unscreened case, the gap $\Delta_{e-h}^{(S)}$ does not depend on the angular momentum of the added electron or hole. 


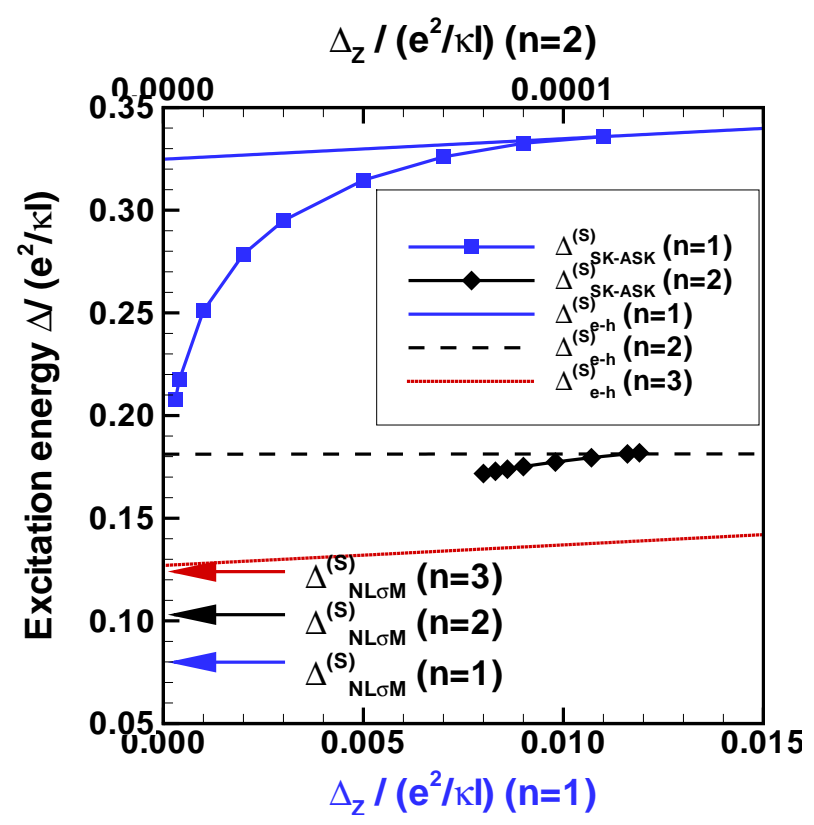

FIG. 7: (Color online) Excitation energy of a Hartree-Fock electron-hole pair $\Delta_{e-h}^{(S)}$ and a skyrmion-antiskyrmion pair $\Delta_{S-a S}^{(S)}$ with screening corrections for Landau levels $n=1$ (lower $x$ axis) and $n=2$ (upper $x$ axis) and $n=3$ (lower $x$ axis) at half-filling. The arrow points to the excitation energy of skyrmion-antiskyrmion pair obtained by $N L \sigma$ model with a screened stiffness.

Fig. 7 shows the energy gaps when screening is taken into account for Landau levels $n=1,2,3$ in the half-filled case. The energy of all three gaps is reduced substantially in comparison with the unscreened results. The value of $\widetilde{g}_{c}$ is also further reduced with respect to its unscreened value. The data points for $n=2$ are not very reliable as they are obtained at very small Zeeman coupling where skyrmions are large. The however provide an upper limit for $\widetilde{g}_{c}$. The transport gap is due to skyrmions for $n=2$ in the screened case but only at very small Zeeman coupling. For Landau level $n=3$, the Zeeman range of coupling where the transport gap is due to skyrmions is further reduced with respect to the $n=2$ case.

Fig. 8] shows the evolution of the $N L \sigma M$ and electronhole transport gaps with Landau level index at half-filling and zero Zeeman coupling. The behavior of the ratios $\Delta_{e-h} / \Delta_{e-h}^{(S)}$ and $\Delta_{N L \sigma M} / \Delta_{N L \sigma M}^{(S)}$ with Landau level index is shown in the inset. The screening corrections saturate at large $n$ more rapidly for skyrmions than for electron-hole pairs. As with $\Delta_{N L \sigma M}$, the gap $\Delta_{N L \sigma M}^{(S)}$ increases with Landau level index but much less rapidly than in the unscreened case.

For completeness, the calculation of the pair energy in both the screened and unscreened cases for Landau level $n=0$ is shown in Fig. 9 and the corresponding number of down spins is shown in Fig. 10 As we remarked above, the exact nature of the ground state for $n=0$ in graphene is still controversial and is probably not spin

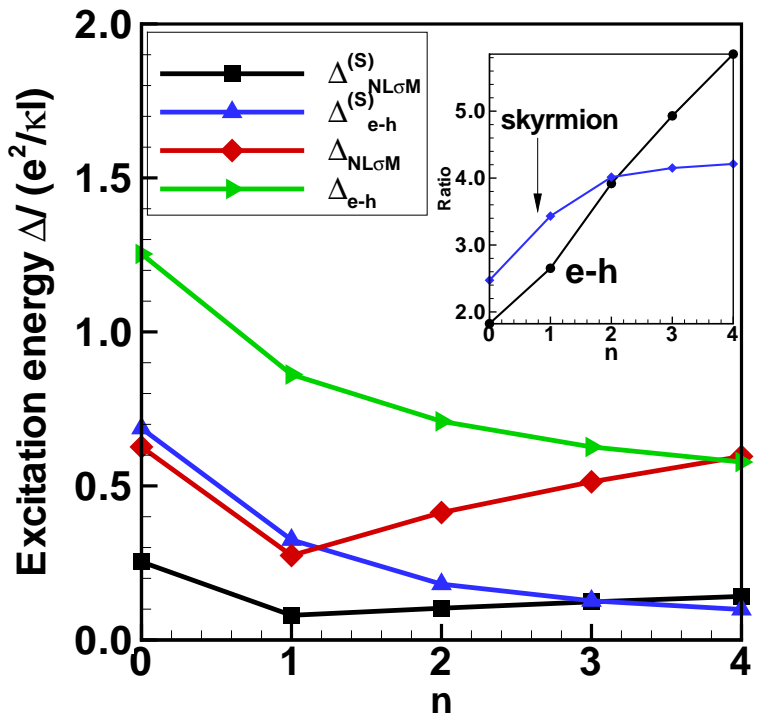

FIG. 8: (Color online) Evolution of the $N L \sigma M$ and electronhole transport gaps at zero Zeeman coupling with Landau levels $n=1,2,3$. The full lines are only a guide to the eyes. The inset shows the ratios $\Delta_{e-h} / \Delta_{e-h}^{(S)}$ and $\Delta_{N L \sigma M} / \Delta_{N L \sigma M}^{(S)}$ with Landau level index.

polarized. Nevertheless, our calculation for $n=0$ is valid for a $\mathrm{S}$-aS pair energy in a conventional 2DEG.

At $1 / 4$ and $3 / 4$ fillings of the Landau levels $|n| \geq 1$, the ground state is valley and spin polarized. Both polarizations are not maximal, however. At sufficiently large Zeeman coupling, spin flips are prohibited and the lowestenergy charged excitations must be valley skyrmions ${ }^{6}$ with up spins at $1 / 4$ filling and down spins at $3 / 4$. Because there is no symmetry breaking term for the valley pseudospin, the $N L \sigma$ model can be used to compute the $\mathrm{S}-\mathrm{aS}$ excitation energy. In the absence of screening, the gap $\Delta_{N L \sigma M}$ for valley skyrmion at $1 / 4$ and $3 / 4$ fillings is identical to that at half-filling shown in Fig. 8, As Fig. 6] indicates, however, screening is more important at $3 / 4$ filling than at $1 / 4$ so that we expect the transport gap to be smaller in the former case. Fig. [1] shows that this is indeed the case in all Landau levels $n$. This conclusion agrees with the experimental results. Although there is a large sample variability in the magnitude of the transport gap due to the different disorders, the gaps measured at $\nu=-5$ are systematically smaller than those measured at $\nu=-3$ (see Fig. 4 of Ref. 2) by a factor $\approx 1.3$ which is close to that measured experimentally. The measurements show only a minimal dependence of the gaps with the perpendicular magnetic field so that our assumption of no spin flip is justified. 


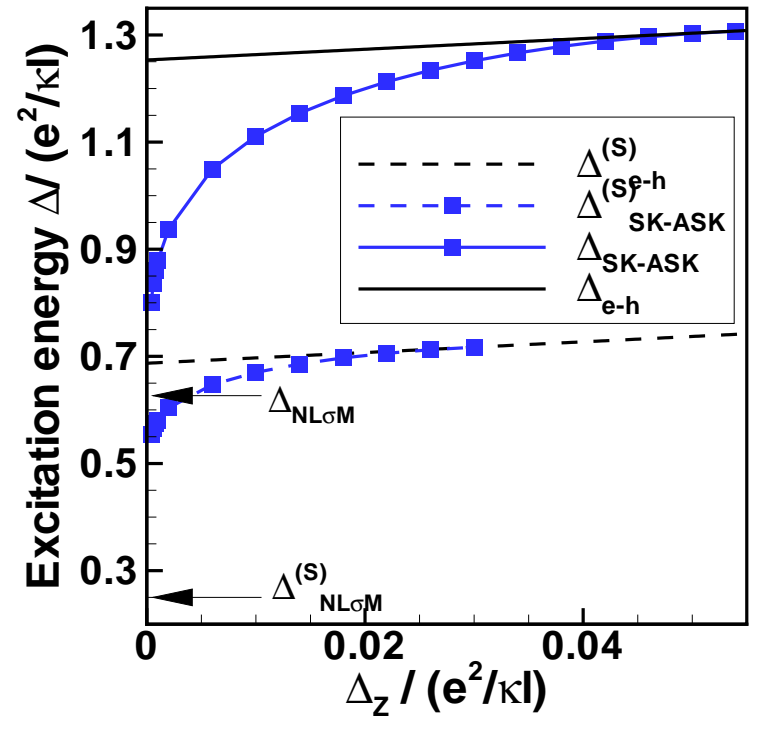

FIG. 9: (Color online) Excitation energy of a Hartree-Fock electron-hole pair and a spin skyrmion-antiskyrmion pair with and without screening corrections for Landau level $n=0$.

\section{CONCLUSION}

We have computed numerically the electron-hole and spin skyrmion-antiskyrmion transport gaps in Landau levels $n=1$ to $n=3$ in graphene as a function of the Zeeman coupling strength. Our calculation used a microscopic wave function for the spin-texture excitations and the energy was computed in the Hartree-Fock approximation. By keeping a large number of orbital momenta (up to $m_{\max }=1000$ ) in the calculation, we were able to obtain the transport gap at very small value of the Zeeman coupling $\widetilde{g} \approx 0.001$.

Previous calculations at zero Zeeman coupling using the nonlinear $\sigma$ mode $^{16}$ already indicated that the transport gap is due to spin texture excitations in graphene at half-filling of the Landau levels $n=1,2,3$ (Landau level $n=0$ is not spin polarized at half-filling) and to valley skyrmions at $1 / 4$ and $3 / 4$ fillings. By comparison, skyrmions are the lowest-energy charged excitations in conventional 2DEG only in Landau level $n=0$. Our calculations confirm the $N L \sigma M$ results and indicate that the spin texture excitations persist for $n=1$ up to $\widetilde{g}_{c} \approx 0.05$ or $\widetilde{g}_{c} \approx 0.011$ when screening corrections are included and up to $\widetilde{g}_{c} \approx 0.0026$ for $n=2$ in the absence of screening. In the screened case for $n=2$ and in both cases for $n=3$, critical value of $\widetilde{g}_{c}$ is very small and a reliable numerical result is difficult to obtain. Skyrmions are the lowest-energy excitations in theses cases only in a very small range of Zeeman coupling.
For valley skyrmions, there is no symmetry-breaking term equivalent to the Zeeman coupling so that the transport gap can be computed using the $N L \sigma M$ if spin flips

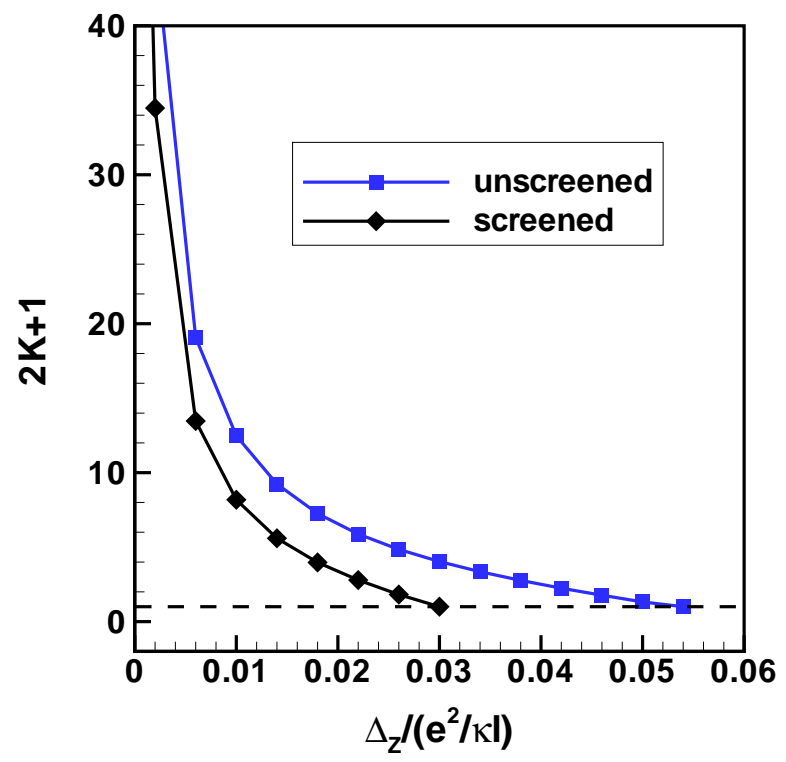

FIG. 10: (Color online) Number of reversed spins $N_{\downarrow}=2 K+1$ in a spin skyrmion-antiskyrmion pair in Landau level $n=0$ as a function of the Zeeman coupling for the screened and unscreened Coulomb interaction. The dashed line indicates $K=0$.

are prohibited by a finite Zeeman coupling. Our results show that screening corrections are more important at $3 / 4$ filling than at $1 / 4$ so that the transport gap due to unbound valley skyrmion-antiskyrmion excitations is smaller in the former case.

Although screening corrections reduce substantially the size of the transport gap, the theoretical value is still large in comparison with the experimental result. Including disorder and Landau level mixing might help to decrease the gap to a more realistic value. We may also consider working with the full four-level model i.e. consider skyrmions with intertwined spin and valley textures.

\section{Acknowledgments}

R. Côté was supported by a grant from the Natural Sciences and Engineering Research Council of Canada (NSERC). Computer time was provided by Calcul Québec and Compute Canada. W. Luo would like to thank Dr. Huizhong Lu of Calcul Québec for helpful discussions. 


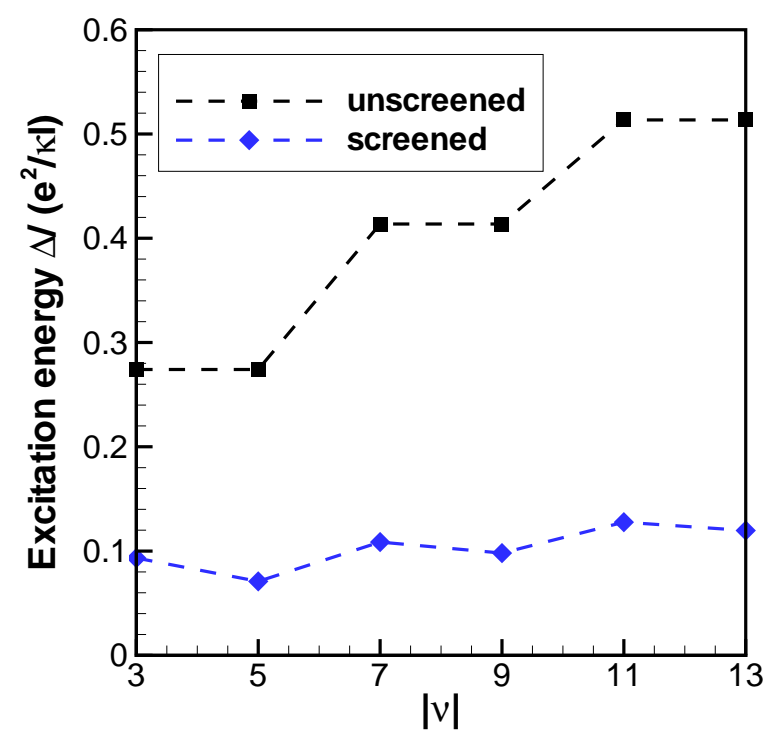

FIG. 11: (Color online) Excitation energy of a valley skyrmion-antiskyrmion pair for different filling factors with and without screening corrections. The dashed lines are only a guide to the eyes.

1 For a review of the properties of graphene, see for example A. H. Castro Neto, F. Guinea, N. M. R. Peres, K. S. Novoselov and A. K. Geim, Rev. Mod. Phys. 81, 109 (2009).

2 A. F. Young, C. R. Dean, L. Wang, H. Ren, P. CaddenZimansky, K. Watanabe, T. Taniguchi, J. Hone, K. L. Shepard and P. Kim, Nature Phys. 8, 550 (2012).

${ }^{3}$ Y. Zhang, Z. Jiang, J. P. Small, M. S. Purewal, Y.-W. Tan, M. Fazlollahi, J. D. Chudow, J. A. Jaszczak, H. L. Stormer, and P. Kim, Phys. Rev. Lett. 96, 136806 (2006).

4 S. L. Sondhi, A. Karlhede, S. A. Kivelson and E. H. Rezayi, Phys. Rev. B 47, 16419 (1993).

${ }^{5}$ For a review of skyrmions in the context of the quantum Hall effect, see for example: Z. F. Ezawa, Quantum Hall Effects (World Scientific, Singapore 2000).

${ }^{6}$ Kun Yang, S. Das Sarma, and A. H. MacDonald, Phys. Rev. B 74, 075423 (2006).

7 I. Mihalek and H. A. Fertig, Phys Rev. B 6213573 (2000).

${ }^{8}$ H. A. Fertig, Luis Brey, R. Côté, A. H. MacDonald, A. Karlhede, and S. L. Sondhi, Phys. Rev. B 55, 10671 (1997).

9 X.-G. Wu and S. L. Sondhi, Phys. Rev. B 51, 14725 (1995).

10 Naokazu Shibata and Kentaro Nomura, Phys. Rev. B 77, 235426 (2008); Naokazy Shibata and Kentaro Nomura, J. Phys. Soc. Jpn. 78, 104708 (2009).

11 Csaba Töke, P. E. Lammert, V. H. Crespi, and J. K. Jain, Phys. Rev. B 74, 235417 (2006).

12 R. Côté, J.-F. Jobidon, and H. A. Fertig, Phys. Rev. B 78, 085309 (2008).

13 B. Douçot, M. O. Goerbig, P. Lederer, and R. Moessner, Phys. Rev. B 78, 195327 (2008).

14 R. Rajaraman, Solitons and Instantons (North-Holland,
Amsterdam, 1989).

15 H. A. Fertig, L. Brey, R. Côté, and A. H. MacDonald, Phys. Rev. B 50, 11018 (1994).

16 D. Yoshioka, The Quantum Hall Effect (Springer-Verlag, Berlin 2002).

17 M. O. Goerbig, R. Moessner, and B. Douçot, Phys. Rev. B 74, 161407(R) (2006).

18 J. G. Checkelsky, L. Li, and N. P. Ong, Phys. Rev. Lett. 100, 206801 (2008); X. Du, I. Skachko, F. Duerr, A. Luican, and E. Y. Andrei, Nature 462, 192 (2009); J. G. Checkelsky, L. Li, and N. P. Ong, Phys. Rev. B 79, 115434 (2009); L. Zhang, J. Camacho, H. Cao, Y. P. Chen, M. Khodas, D. E. Kharzeev, A. M. Tsvelik, T. Valla, and I. A. Zaliznyak, Phys. Rev. B 80, 241412 (2009); L. Zhang, Y. Zhang, M. Khodas, T. Valla, and I. A. Zaliznyak, Phys. Rev. Lett. 105, 046804 (2010).

19 K. Moon, H. Mori, Kun Yang, S. M. Girvin, A. H. MacDonald, L. Zheng, D. Yoshioka, Shou-Cheng Zhang, Phys. Rev. B 51, 5138 (1995).

20 A. H. MacDonald and S. M. Girvin, Phys. Rev. B 34, 5639 (1986).

21 S. Melinte, E. Grivei, V. Bayot, and M. Shayegan, Phys. Rev. Lett. 82, 2764 (1999).

${ }^{22}$ Norio Kumada, Anju Sawada, Zyun F. Ezawa, Satoshi Nagaham, Hirofumi Azuhata, Koji Muraki, Tadashi Saku, and Yoshiro Hirayama, J. Phys. Soc. Jpn. 69, 3178 (2000).

23 I. L. Aleiner and L. I. Glazman, Phys. Rev. B 52, 11296 (1995).

24 The screening of the Fock term only, when the dielectric function is evaluated using the Lindhard function with the non-interacting energy levels, leads to unphysical results. 
25 Michael M. Fogler in High Magnetic Fields: Applications in Condensed Matter Physics and Spectroscopy, ed. by C. Berthier, L.-P. Levy, G. Martinez (Springer-Verlag, Berlin 2002).
26 K. Shizuya, Phys. Rev. B 75, 245417 (2007) and R. Roldan, M. O. Goerbig and J.-N. Fuchs, Semicond. Sci. Technol. 25, 034005 (2010). 